\author{
CHAPTER 9
}

\title{
Neural stem and progenitor cells: choosing the right Shc
}

\author{
Tiziana Cataudella, Luciano Conti and Elena Cattaneo* \\ Department of Pharmacological Sciences and Center of Excellence on Neurodegenerative Diseases, \\ University of Milan, I-20133 Milan, Italy
}

\begin{abstract}
Neural stem cell (NSCs) are self-renewing, multipotent cells able to generate neurons, astrocytes and oligodendrocytes. Since their identification, these properties have made NSCs an attractive subject for therapeutic applications to the damaged brain. In this context, understanding the mechanisms and the molecules regulating their biological properties is important and it is focused to gain control over their proliferative and differentiative potential. Here we will discuss values and unsolved aspects of the system and the employment of potentially key molecular targets for proper control of NSCs fate.
\end{abstract}

Keywords: self-renewal; neuronal production; signaling proteins; progenitors

\section{Introduction}

Throughout the last decade there has been an increasingly enthusiastic interest in the cell biology of stem cells. These are widely considered as an invaluable potential tool for cell therapy approaches to a broad range of clinical conditions. According to its definition, the main physiologic function of a stem cell is to generate all of the differentiated cell types of the tissue in which it resides. Indeed, a stem cell is generally defined operationally as a cell that is: (i) multipotent, (ii) capable of self-renewal, and (iii) capable of generating a progeny that can functionally integrate into and repair the tissue of origin.

Regarding the neural stem cells (NCSs), i.e., those stem cells residing inside the nervous system, this implies that their progeny will include mature neurons, astrocytes and oligodendrocytes. Stem cell technology is particularly important for the central

*Correspondence to: E. Cattaneo, Department of Pharmacological Sciences and Center of Excellence on Neurodegenerative Diseases, University of Milan, Via Balzaretti 9, I-20133 Milan, Italy. Tel.: + 39-02-5031-8333; Fax:+39-02-5031-8284; E-mail: elena.cattaneo@unimi.it nervous system (CNS) since cell transplantation might help to overcome the intrinsic poor capability of the nervous tissue to replace elements lost in the course of injury or disease. Proper control over the differentiation pattern of brain stem cells may therefore eventually allow the treatment of a wide range of degenerative diseases characterized by neuronal or glial loss. However, relatively little is known about the molecular regulators and genetic cascades that control NSCs self-renewal and multipotency. In this article, we will review the recent advancements in the NSCs field aimed to improve the control of their proliferation or differentiation, aiming at an efficient and safe future clinical employment for cell replacement purposes.

\section{Stem or progenitor cells: how to distinguish between the two?}

Cells with stem-like properties, initially identified in the fetal, and more recently in the adult mammalian brain, can be grown in culture, displaying the potential to self renew and to generate the different 
cell types of the nervous system (McKay, 1997). During brain development, NSCs are localized in the epithelial layer of the germinal zone surrounding the ventricles (Temple, 2001). As brain maturation continues, postmitotic neurons migrate away from the ventricular zone, mainly guided by radially oriented glial processes, and the ventricular zone diminishes in size (Rao, 1999). In the adult brain, cells with similar stem-like properties also exist, mostly originating from two regions: the hippocampus and the subventricular zone (SVZ) of the lateral ventricles (Gage, 2002). Noteworthy, different studies indicate that NSCs from different fetal and adult brain areas are not identical, as demonstrated by different growth characteristics, trophic factor requirements, and specific patterns of differentiation (Temple, 2001). This is further demonstrated by in vitro experiments that revealed that NSCs differ in their potential according to the developmental stage at which they are isolated and to the site from where they were obtained (Temple, 2001). More generally, in vitro studies indicate that two types of neural stem-like cells have been isolated in multiple brain regions and appear to coexist. One type shows epidermal growth factor (EGF) responsiveness and can be expanded as floating cell aggregates, called neurospheres (Reynolds and Weiss, 1992), becoming fibroblast growth factor (FGF)-responsive with the in vitro passages (Vescovi et al., 1993; Represa et al., 2001). The second group has been shown to be FGF-dependent and can be propagated both as adherent cultures as well as neurospheres (Kalyani et al., 1997).

Importantly, all the above mentioned evidence indicating the existence of heterogeneous NSC populations may be the consequence of the lack of a neural stem-restricted marker for NSC prospective isolation procedure. This implies that it is currently very difficult to distinguish, but posteriorly and only following accurate clonal analysis, between a real NSC and a progenitor. Conceptually, these two populations differ for their differentiative capabilities. Indeed, while NSCs are multipotential, brain progenitor are generally considered to be more limited in their potential and able to produce only restricted phenotypes (McKay, 1997). Up to date, there are only few markers of putative NSCs (nestin, sox-1, musashi, AC133, $\mathrm{PNA}^{\text {low }} / \mathrm{HSA}^{\text {low }}$, Lex/ssea-1) that may be used with some degree of specificity being expressed by brain progenitors and not by other cell types (Rossi and Cattaneo, 2002).

In the absence of a clear in vivo assay to identify the NSCs, most authors use the ability to grow, in vitro, neurospheres which contain cells capable to differentiate as glia and neurons, as an operative definition for 'NSCs' (Rossi and Cattaneo, 2002). Nevertheless, this assay is not devoid of faults since neurospheres contain cells that are clearly not uniform in their differentiative stage and estimation of the number of bona fide stem cells contained in a preparation of cells dissociated from neurospheres varies as widely as for those contained in the fetal brain (Temple, 2001; Rossi and Cattaneo, 2002; Suslov et al., 2002). New strategies to allow efficient prospective isolation of stem cells from the brain are highly demanded to guarantee uniformity of results and lead to trustworthy conclusions. Increasing attempts in this direction are ongoing by combining sorting for cell size and antigenic properties (Rietze et al., 2001; Capela and Temple, 2002).

\section{Transdifferentiation, transformation, fusion: deciphering the stem cells plasticity}

Recent progress in stem cell research indicates that certain mammalian cells maintain a high degree of plasticity giving rise to multilineage cell differentiation. Indeed, some developmental peculiarities suggest that stem cells may be able to differentiate into cell types that are not of the same germ layers (Tajbakhsh et al., 1994). An early intriguing case is that of cultured neural stem cells derived from neurospheres of clonogenic origin which repopulate the hematopoietic system in sublethally irradiated allogenic host by cells (Bjornson et al. 1999). Similarly, in other studies, transplantation of adult bone marrow cells has generated a broad range of phenotypes, including muscle cells (Ferrari et al., 1998), liver cells (Petersen et al., 1999; Lagasse et al., 2000), brain cells (Brazelton et al., 2000; Mezey et al., 2000) and other (Krause et al., 2001). This seemed to indicate that the extracellular factors or cell-cell interaction might be sufficient for reprogramming putative somatic stem cells into a more pluripotent, embryonic stem cell (ES)-like, condition. This hypothesis was reinforced by the demonstration that when injected 
into blastocysts NSCs participate to the formation of most of the tissues of the mouse (Clarke et al., 2000). This hypothesis is challenged by results from various groups. Morshead and colleagues showed that, in their hands, putative NSCs extensively cultivated in vitro do not turn into hematopoietic cells with any appreciable frequence (Morshead et al., 2002). The same authors suggested that genetic instability of NSCs after long term in vitro expansion may explain the original results. They suggested that the claimed hematopoietic reconstitution could result as a consequence of transformation events or artefacts due to the in vitro procedures or methodological problems. While this study was criticized for the possible different number of stem cells in the culture and the presence of transformation events (Vescovi et al., 2002), it seems reasonable to conclude that transdifferentiation events, when present, appears to be a much rarer phenomenon than previously described and may be peculiar only to some cell clones. Because of that, the clinical relevance of such data is debated. Nevertheless, the possibility that even a few brain or blood stem cells can transdifferentiate remains of biological interest. However, no consistent demonstration of cell conversion followed the original claims, leaving the subject, at present, rather confused.

Following the report of Bjornson et al. (1999), we began a set of experiments aimed at assessing whether the reported ability of NSCs to transdifferentiate into blood was acquired due to in vitro expansion (and possible de-differentiation of the cells), or whether it was an intrinsic property of brain stem cells (Magrassi et al., 2003). To test this, we isolated fetal neural cells (fNC), from E10 embryos derived of transgenic mice expressing EGFP (at this stage the telencephalic vesicles do not have ingrowths of blood vessels and are highly enriched in NSCs and progenitors). The cells were dissociated and directly transplanted into sublethally irradiated $\mathrm{C} 57 \mathrm{Bl} / 6$ as performed by Bjornson et al. (1999). Analysis of grafted animals at different time points did not reveal development of chimerism in the hematopoietic compartment even after very long survival times (16 months) after grafting. The same negative results were obtained by injecting the $\mathrm{fNC}$ into the tail vein or by transplanting them directly into the bone marrow cavity of sublethally irradiated mice. While we could detect donor cells by PCR and Fluorescence Activated Cell Sorting (FACS) analyzes at the early time points, the same assays failed to detect the presence of circulating donor-derived fNC EGFP positive cell on a fraction of peripheral blood collected from the grafted animals at mid and at late time points (i.e., 30, 60, 120 and 495 days after grafting) (Magrassi et al., 2003). These results indicated that NSCs do not physiologically exhibit transdifferentiative capability and that, if present, this capability requires ex vivo expansion procedures that would possibly reprogramme the differentiative potential of the donor cells. This seems in agreement with recent results from Verfaille's group that has reported the isolation of a Multipotent Adult Progenitor cell (MAPc) able to extensively contribute to chimerae once injected in the early blastocyst and to repopulate all the adult tissues once transplanted into sublethally irradiated mice (Jiang et al., 2002). Interestingly, these cells can be established from adult murine mesenchymal cells only after at least 20 passages in vitro, reinforcing the idea that the in vitro expansion may somehow act to reprogram the cells toward an ES-like potential.

However, various studies now indicate that transdifferentiation of in vitro expanded stem cells may be explained by fusion of donor cells with host cells (Terada et al., 2002; Ying et al., 2002). While these studies were showing the possibility of cell fusion between somatic stem cells and cultured embryonic stem cells, more recent experiments now indicate the possibility that cell fusion may also occur among somatic tissues after in vivo transplantation (Vassilopoulos et al., 2003; Wang et al., 2003).

Future research is needed to establish the real ability of stem cells to transdifferentiate and the impact of this phenomena for therapeutical approaches.

\section{Driving proliferation and differentiation events in neural cells through Shc(s) molecules}

The identification of candidate molecular mechanisms able to modulate proliferation, differentiation and, possibly, plasticity of NCSs is of great interest in order to implement studies and trials for cell replacement therapies. To this regard, an interesting strategy may come from studies of signaling 
mechanisms downstream of growth factors receptors. Particularly, we pointed to the regulated expression and activity of Shc(s) adapter molecules, which couple signals from activated receptors to downstream effectors, as a potential mechanism to regulate division, survival and differentiation on stem cells in the brain. Shc(s) proteins indeed appear to play a role in the control of the proliferation and subsequent maturation of mitotically active neural stem/progenitor cells into postmitotic neurons (Cattaneo and Pelicci, 1998; Conti et al., 2001).

Up to date, three $\mathrm{Shc}(\mathrm{s})$ genes have been identified, named ShcA, ShcB/Sli and ShcC/Rai/N-Shc having a consistent homology (Pelicci et al., 1992, 1996; O’bryan et al., 1996; Luzi et al., 2000). These three Shc(s) molecules are characterized by the presence of phosphotyrosine regulatory residues and the PTB, CH1 (a proline rich domain) and SH2 domains in the presented order. Three isoforms are known for ShcA (of 66, 52 and $46 \mathrm{kD}$ ), two isoforms for ShcB (of 52 and $47 \mathrm{kD}$ ) and two for ShcC (of 54 and $69 \mathrm{kD}$ ). $\mathrm{p} 6^{\text {ShcA }}$ display a further $\mathrm{N}$-terminal $\mathrm{CH}$ domain $(\mathrm{CH} 2)$ that contains important regulatory serine residues. They share elevated homology in both the $\mathrm{C}$ terminus $\mathrm{SH} 2$ domain and the $\mathrm{N}$ terminus PTB domain, the most divergent sequence being in the proline and glycine rich $\mathrm{CH} 1$ (Collagene Homology 1) region. ShcA proteins have been extensively characterized and shown to be widely expressed outside the CNS. Their importance is indicated by (i) the early embryonic lethal phenotype

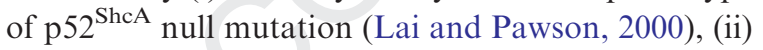
the impairment in thymocytes development in conditional $\mathrm{p}^{52^{\text {ShcA }}}$ knockout (Zhang et al., 2002), and (iii) by the increase in life span and resistance

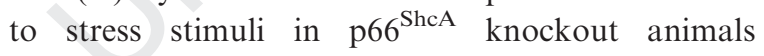
(Migliaccio et al., 1999).

Despite the apparently constitutive presence of ShcA in extraneural tissues, ShcA expression and activity within the brain is tightly regulated during development and maximal in the embryonic day 10 neural tube. At later time points, ShcA remains confined to the germinal epithelium where mitotically active immature stem and progenitor cells are located. Instead, in the areas of the embryonic or postnatal brain where postmitotic neurons are present, mRNA $^{\text {ShcA }}$ is highly reduced. Similarly, the adult brain exhibited low ShcA expression, the main exception being the olfactory epithelium, which is a predominant area of active neurogenesis in the adult. These changes in the expression and activity of ShcA as a function of neuronal maturation were confirmed in vitro in differentiating neuronal cultures (Conti et al., 1997). Not only ShcA is present in actively dividing neurogenic areas, but is also susceptible of being activated. Indeed, in vivo immunoprecipitation of ShcA from the telencephalic vesicles of embryonic brains injected intraventricularly with mitogens like EGF revealed a higher phosphorylation of the $\mathrm{p} 52^{\text {ShcA }}$ isoform with respect to control animals (Conti et al., 1997). In treated samples, Grb2 coimmunoprecipitation was also observed, indicating that ShcA is not only present in the germinal epithelium, but it is also able to elicit a functional response with recruitment of downstream pathways.

The demonstration that ShcA availability is regulated during neurogenesis and becomes limited during NSCs maturation in vivo and in vitro led to the proposition that other Shc-like proteins may substitute for ShcA function in mature neurons (Cattaneo and Pelicci, 1998). Given the existence of two more recently identified Shc members, ShcB and $\mathrm{ShcC}$, the latter being selectively expressed in the brain, we suggested that one or both of them could replace ShcA in mature neurons (Cattaneo and Pelicci, 1998). Analyzes of ShcC expression showed an opposite expression pattern with respect to ShcA, being absent in neural progenitors but present in early postmitotic neurons and reaching maximal levels in the adult brain where it is found localized only in neurons. Similar changes in ShcA and ShcC levels during neuronal maturation have been observed in several mammalian species (rat, mouse and human) (Conti et al., 2001). Notably, $\mathrm{ShcC}$ is found in neurons from various regions of the adult brain thus predicting a general role played by ShcC in these cells. Particularly, given the above described central roles of ShcA in signal transduction, ShcC appearance in differentiating NSCs has been hypothesized to serve different 'connector functions' compared with ShcA, allowing maturing cells to respond differently to environmental stimuli (Conti et al., 2001). To this regard, Lai and Pawson (2000) demonstrated the existence of a strict link between Shc levels and cell responsiveness. The authors showed that ShcA expression and activity are 
required in cells of the cardiovascular system to make them responsive to low concentrations of growth factors. Indeed, while a low concentration of growth factors is necessary to activate the MAPK pathway in mouse embryo fibroblasts (MEF), cells from ShcA knockout mice require a higher concentration of growth factors to activate the same signaling cascade. Transfection experiments in primary neural cells and in postmitotic neurons revealed that ShcC acts to promote neuronal differentiation and improve survival of these cells (Conti et al., 2001). It was also found that ShcC elicits these effects through a different kinetic of activation of downstream effector molecules with respect to ShcA. Indeed, ShcC elicits neuronal differentiation via prolonged stimulation of the MAPK (Conti et al., 2001; Pelicci et al., 2002). This behavior is reminiscent of that described in PC12 cells exposed to NGF, where persistent activation of MAPK is required for neuronal differentiation. On the contrary, ShcC-driven prosurvival effect occurs via recruitment of the PI3K-Akt pathway (Conti et al., 2001; Pelicci et al., 2002), as demonstrated by the fact that its pharmacological or molecular inhibition markedly abolishes this effect. To this respect, ShcC-induced Akt activation was found to cause phosphorylation (with inhibition) of $\mathrm{Bad}$, a proapoptotic member of the $\mathrm{Bcl} 2$ family (Conti et al., 2001).

Single and double $S h c B / C$ null mice have been recently described (Sakai et al., 2000). ShcB-deficient mice exhibit a loss of peptidergic and nonpeptidergic nociceptive sensory neurons. ShcC null mice appear not to show gross anatomical abnormalities. Noteworthy, mice lacking both ShcB and ShcC exhibit a significant additional loss of neurons within the superior cervical ganglia. This aspect may emphasize that the lack of phenotype in ShcC null mice could be due to a partial compensation by the other ShcB or other Shc members during development, thus masking ShcC real function in neural tissues. Further analyzes will be required to elucidate ShcC role in neuronal generation from stem cells .

Taken together these results unveil a new scenario within which physiological changes in the availability of ShcA and ShcC adaptors during brain development may act to modify neural stem/progenitor cell responsiveness as a function of the new and developing environment.

\section{Conclusions}

Fetal and adult NSCs are an important tool to be exploited for brain repair in neurodegenerative disease either through their transplantation or via their in situ activation in the brain. The understanding of the function of candidate genes responsible for proliferation and differentiation is essential to implement studies for cell replecement therapies; however, at present, the specification of NSC into the desired phenotypes is far from being efficiently controlled. The prominent activities of Shc(s) proteins in modulating cell responsiveness and the demonstration of their regulated expression at the transition from proliferation to differentiation in the brain, point at the Shc(s) signaling pathways as candidate targets for pharmacological modulation of stem cell division and differentiation in the brain.

\section{Acknowledgments}

The work of the authors described in this article is supported by grants from the Ministry of Research and University (MIUR, 2001055212-004), by a F.I.R.B. National Research Network on Neural Stem Cells coordinated by E.C. (MIUR, RBNE01YRA31), Telethon Onlus (\#GP0215Y02), and Associazione Italiana Ricerca sul Cancro (AIRC, Italy) to E.C. and Telethon Onlus (\#GGPO2457) to L.C.
Abbreviations
NSCs neural stem cells
CNS central nervous system
SVZ subventricular zone
EGF epidermal growth factor
FGF fibroblast growth factor
EC embryonic stem cell
fNC fetal Neural stem and progenitor Cells
EGFP enhanced green fluorescent protein
FACS fluorescence activated cell sorting
MAPc multipotent adult progenitor cell 


$\begin{array}{lll}241 & \text { Shc } & \text { Src Homologue and Collagene } \\ 242 & & \text { Homologue } \\ 243 & \text { PNA } & \text { peanut agglutinin } \\ 244 & \text { HAS } & \text { heat-stable antigen } \\ 245 & \text { LeX } & \text { Lewis X } \\ 246 & \text { SSEA-1 } & \text { stage-specific embryonic antigen 1 }\end{array}$

\section{References}

Bjornson, C.R., Rietze, R.L., Reynolds, B.A., Magli, M.C. and Vescovi, al. (1999) Turning brain into blood: a hematopoietic fate adopted by adult neural stem cells in vivo. Science, 283: 534-537.

Brazelton, T.R., Rossi, F.M.V., Keshet, G.I. and Blau, H.M. (2000) From marrow to brain: expression of neural phenotypes in adult mice. Science, 290: 1775-1779.

Capela, A. and Temple, S. (2002) LeX/ssea-1 is expressed by adult mouse CNS stem cells, identifying them as nonependymal. Neuron, 35: 865-875.

Cattaneo, E. and Pelicci, P.G. (1998) Emerging roles for Sh2/ PTB-containing Shc adapter proteins in the developing mammalian brain. Trends Neurosci., 21: 476-481.

Clarke, D.L., Johansson, C.B., Wilbertz, J., Veress, B., Nilsson, E., Karlstrom, H., Lendahl, U. and Frisen, J. (2000) Generalized potential of adult neural stem cells. Science, 288: 1660-1663.

Conti, L., De Fraja, C., Gulisano, M., Migliaccio, E., Govoni, S. and Cattaneo, E. (1997) Expression and activation of SH2/PTB-containing ShcA adaptor protein reflects the pattern of neurogenesis in the mammalian brain. Proc. Natl. Acad. Sci. USA, 94: 8185-8190.

Conti, L., Sipione, S., Magrassi, L., Bonfanti, L., Rigamonti, D., Pettirossi, V., Peschanski, M., Haddad, B., Pelicci, P., Milanesi, G., Pelicci, G. and Cattaneo, E. (2001) She signaling in differentiating neural progenitor cells. Nat. Neurosci., 4: 579-586.

Ferrari, G., Cusella-De Angelis, G., Coletta, M., Paolucci, E., Stornaiuolo, A., Cossu, G. and Mavilio, F. (1998) Muscle regeneration by bone marrow-derived myogenic progenitors. Science, 279: 1528-1530.

Gage, F.H. (2000) Mammalian neural stem cells. Science, 287: 1433-1438.

Gage, F.H. (2002) Neurogenesis in the adult brain. J. Neurosci., 22: 612-613.

Jiang, Y., Jahagirdar, B.N., Reinhardt, R.L., Schwartz, R.E., Keene, C.D., Ortiz-Gonzalez, X.R., Reyes, M., Lenvik, T., Lund, T., Blackstad, M., Du, J., Aldrich, S., Lisberg, A., Low, W.C., Largaespada, D.A. and Verfaillie, C.M. (2002) Pluripotency of mesenchymal stem cells derived from adult marrow. Nature, 4418: 41-49.
Kalyani, A., Hobson, K. and Rao, M.S. (1997) Neuroepithelial stem cells from the embryonic spinal cord: isolation, characterization, and clonal analysis. Dev. Biol., 186: 202-223.

Krause, D.S., Theise, N.D., Collector, M.I., Henegariu, O., Hwang, S., Gardner, R., Neutzel, S. and Sharkis, S.J. (2001) Multi-organ, multi-lineage engrafment by a single bone marrow-derived stem cell. Cell, 105: 369-377.

Lagasse, E., Connors, H., Al-Dhalimy, M., Reitsma, M., Dohse, M., Osborne, L., Wang, X., Finegold, M., Weissman, I.L. and Grompe, M. (2000) Purified hematopoietic stem cells can differentiate into hepatocytes in vivo. Nat. Med., 6: 1229-1234.

Lai, K.M.V. and Pawson, T. (2000) The ShcA phosphotyrosine docking protein sensitizes cardiovascular signaling in the mouse embryo. Genes Dev., 14: 1132-1145.

Luzi, L., Confalonieri, S., DiFiore, P.P. and Pelicci, P.G. (2000) Evolution of She functions from nematode to human. Curr. Opin. Genet. Dev., 10: 668-674.

Magrassi, L., Castello, S., Ciardelli, L., Podesta', M., Gasparoni, A., Conti, L., Pezzotta, S., Frassoni, F. and Cattaneo, E. (2003) Freshly dissociated fetal neural stem/ progenitor cells do not turn into blood. Mol. Cell. Neurosci., 22: 179-187.

McKay, R. (1997) Stem cells in the central nervous system. Science, 276: 66-71.

Mezey, E., Chandross, K.J., Harta, G., Maki, R.A. and McKercher, S.R. (2000) Turning blood into brain: cells bearing neuronal antigens generated in vivo fron bone marrow. Science, 290: 1779-1782.

Migliaccio, E., Giorgio, M., Mele, S., Pelicci, G., Reboldi, P., Panolfi, P.P., Lanfrancone, L. and Pelicci, P.G. (1999) The p66shc adaptor protein controls oxydative stress response and life span in mammals. Nature, 402: 309-313.

Mokry, J., Subrtova, D. and Nemecek, S. (1996) Differentiation of epidermal growth factor-responsive neural precursor cells within neurospheres. Acta Medica (Hradec. Kralove), 39: 7-20.

Morshead, C.M., Benveniste, P., Iscove, N.N. and Van der Kooy, D. (2002) Hematopoietic competence is a rare property of neural stem cells that may depend on genetic and epigenetic alterations. Nat. Med., 8: 268-273.

O’bryan, J.P., Songyang, Z., Cantley, L., Der, C.J. and Pawson, T. (1996) A mammalian adapter protein with conserved src homology 2 and phosphotyrosine binding domains is related to Shc and is specifically expressed in the brain. Proc. Natl. Acad. Sci. USA, 93: 2729-2734.

Pelicci, G., Lanfrancone, L., Grignani, F., McGlade, J., Cavallo, F., Forni, G., Nicoletti, I., Grignani, F., Pawson, T. and Pelicci, P.G. (1992) A novel trasforming protein (Shc) with an $\mathrm{SH} 2$ domain is implicated in mitogenic signal trasduction. Cell, 70: 93-104.

Pelicci, G., Dente, L., De Giuseppe, A., Verducci-Galletti, B., Giuli, S., Mele, S., Vetriani, C., Giorgio, M., Pandolfi, P.P., 
Cesareni, G. and Pelicci, P.G. (1996) A family of Shc related proteins with conserved $\mathrm{PTB}, \mathrm{CH} 1$ and $\mathrm{SH} 2$ regions. Oncogene, 13: 633-641.

Pelicci, G., Troglio, F., Bodini, A., Melillo, R.M., Pettirossi, V., Coda, L., DeGiuseppe, A., Santoro, M. and Pelicci, P.G. (2002) The neuron-specific Rai (ShcC) adaptor protein inhibits apoptosis by coupling Ret to the Phosphoatidylinosiyol 3-kinase/AKT pathway. Mol. Cell. Biol., 22: 7351-7363.

Petersen, B.E., Bowen, W.C., Patrene, K.D., Mars, W.M., Sullivan, A.K., Murase, N., Boggs, S.S., Greenberger, J.S. and Goff, J.P. (1999) Bone marrow as a potential source of hepatic oval cells. Science, 284: 1168-1170.

Rao, M.S. (1999) Multipotent and restricted precursors in the central nervous system. Anat. Rec., 257: 137-148.

Represa, A., Shimazaki, T., Simmonds, M. and Weiss, S. (2001) EGF-responsive neural stem cells are a transient population in the developing mouse spinal cord. Eur. J. Neurosci., 14: 452-462.

Reynolds, B.A. and Weiss, S. (1992) Generation of neurons and astrocytes from isolated cells of the adult mammalian central nervous system. Science, 255: 1707-1710.

Rietze, R.L., Valcanis, H., Brooker, G.F., Thomas, T., Voss, A.K. and Bartlett, P.F. (2001) Purification of a pluripotent neural stem cell from the adult mouse brain. Nature, 412: 736-739.

Rossi, F. and Cattaneo, E. (2002) Opinion: neural stem cell therapy for neurological diseases: dreams and reality. Nat. Rev. Neurosci., 3: 401-409.

Sakai, R., Henderson, J.T., O'Bryan, J.P., Elia, A.J., Saxton, T.M. and Pawson, T. (2000) The mammalian ShcB and ShcC phosphotyrosine docking proteins function in the maturation of sensory and sympathetic neurons. Neuron, 28: 819-833.
Suslov, O.N., Kukekov, V.G., Ignatova, T.N. and Steindler, D.A. (2002) Neural stem cell heterogeneity demonstrated by molecular phenotyping of clonal neurospheres. Proc. Natl. Acad. Sci. USA, 99: 14506-14511.

Tajbakhsh, S., Vivarelli, E., Cusella-De Angelis, G., Rocancourt, D., Buckingham, M. and Cossu, G. (1994) A population of myogenic cells derived from the mouse neural tube. Neuron, 13: 813-821.

Temple, S. (2001) The development of neural stem cells. Nature, 414: 112-117.

Terada, N., Hamazaki, T., Oka, M., Hoki, M., Mastalerz, D.M., Nakano, Y., Meyer, E.M., Morel, L., Petersen, B.E. and Scott, E.W. (2002) Bone marrow cells adopt the phenotype of other cells by spontaneous cell fusion. Nature, 416: 542-545.

Vassilopoulos, G., Wang, P.R. and Russell, D.W. (2003) Transplanted bone marrow regenerates liver by cell fusion. Nature, in press.

Vescovi, A.L., Reynolds, B.A., Fraser, D.D. and Weiss, S. (1993) bFGF regulates the proliferative fate of unipotent (neuronal) and bipotent (neuronal/astroglial) EGF-generated CNS progenitor cells. Neuron, 11: 951-966.

Vescovi, A.L., Rietze, R., Magli, M.C. and Bjornson, C. (2002) Hematopoietic potential of neural stem cells. Nat. Med., 8: 535.

Wang, X., Willenbring, H., Akkari, Y., Torimaru, Y., Foster, M., Al-Dhalimy, M., Lagasse, E., Finegold, M., Olson, S. and Grompe, M. (2003) Cell fusion is the principal source of bone-marrow-derived hepatocytes. Nature, in press.

Ying, Q.L., Nichols, J., Ewans, E.P. and Smith, A.G. (2002) Changing potency by spontaneous fusion. Nature, 416: 545-548.

Zhang, W., Camerini, V., Bender, T.P. and Ravichandran, K.S. (2002) A nonredundant role for the adapter protein Shc in thymic T cell development. Nat. Immunol., 3: 749-755. 
